Bond University

Research Repository

\title{
A model for a reliable single allocation hub network design under massive disruption
}

Yahyaei, Mohsen; Bashiri, Mahdi; Randall, Marcus

Published in:

Applied Soft Computing

DOI:

10.1016/j.asoc.2019.105561

\section{Licence:}

CC BY-NC-ND

Link to output in Bond University research repository.

Recommended citation(APA):

Yahyaei, M., Bashiri, M., \& Randall, M. (2019). A model for a reliable single allocation hub network design under massive disruption. Applied Soft Computing, 82, [105561]. https://doi.org/10.1016/j.asoc.2019.105561

\footnotetext{
General rights

Copyright and moral rights for the publications made accessible in the public portal are retained by the authors and/or other copyright owners and it is a condition of accessing publications that users recognise and abide by the legal requirements associated with these rights.

For more information, or if you believe that this document breaches copyright, please contact the Bond University research repository coordinator.
} 


\title{
A Model for a Reliable Single Allocation Hub Network Design under Massive Disruption
}

\author{
Mohsen Yahyaei \\ Department of Industrial Engineering, Iran University of Science and Technology, \\ Tehran, Iran. \\ Mahdi Bashiri \\ Department of Industrial Engineering, Shahed University, Tehran, Iran. \\ School of science, RMIT University, Melbourne, Australia. \\ Marcus Randall \\ Bond Business School, Bond University, Queensland, Australia.
}

\begin{abstract}
Hub facilities may fail to operate in networks because of accidental failures such as natural disasters. In this paper, a quadratic model was presented for a reliable single allocation hub network under massive random failure of hub facilities which more than one hub may be disrupted in a route. It determines the location of hub facilities and the primal allocation of non-hub nodes. It also determines the backup allocation in case of failure of the primal hub. A lexicographic form of bi-objective quadratic model is developed where the first objective (maximization of served demand or equivalently, minimization of lost flows) must be attained before the second objective (total cost). By adding a structure- based constraint and using enhanced linearization techniques the model was converted into a classic linear zero-one mixed integer model. The applied linear technique was compared with other techniques in terms of computational time and its better performance was approved in problem instances. An iterated local search algorithm was developed to solve large sized instances in reasonable computational time and the compu-
\end{abstract}

\footnotetext{
*Mahdi Bashiri

Email addresses: Mohsen_Yahyaei@ind.iust.ac.ir (Mohsen Yahyaei), Bashiri@shahed.ac.ir (Mahdi Bashiri), Mrandall@bond.edu.au (Marcus Randall)
} 
tational results confirm efficiency of the proposed heuristic. Moreover, the proposed model was compared to the classical hub network using a network performance measure, and the results show the increased efficiency of the model.

Keywords: Hub Location, Disruption, Reliable Network Design, Linearization, Heuristic Algorithm

\section{Introduction}

In facility location problems, network designers try to locate facilities to achieve benefits such as reducing transportation costs and increasing market capture. This requires reliable decisions, so consideration of disruption states appear to be necessary for sustainable location decisions. Hub location [1] is an important applied problem in facility location. It emphasizes the design of compact networks instead of direct origin-destination network connections. In a compact network, hub facilities are intermediate points through which network demands are transported. Because of consolidation effects in hub facilities, network managers benefit from economies of scale. In other words, total transportation costs will decrease when hub points in networks are used. These are also referred to as hub and spoke networks. A good example of this type of network is that used by airlines [2]. It is often the case that regional airports are connected to one or more major (hub) airports. In turn, these hub airports on the whole are all connected to one another and flights between them account for bulk passenger movement.

There are many ways in real life that a part of a hub network can be disrupted. As an example, recent massive snowstorm Jonas, canceled more than 10000 flights and shut down the eastern airspace of the United States. Jonas shut down air transportation hubs such as Washington Dulles and Newark Liberty International Airports [3]. In this paper, we address a single allocation hub location network under hub facility failure probabilities so that temporary diversions can be made while ensuring minimal impact on the objective functions. Massive disruption (simultaneous disruption of both hubs in a route) can occur within the network, and the model tries to tackle it by providing a realistic alternative route for each flow through the network. First, a lexicographic quadratic bi-objective model is developed which the objectives are to minimize of the number of lost flows due to disruption and to minimize network cost. Then, to reduce computational effort of bi-objective 
model, the model is transferred into single one by adding the first objective as structure-based constraint. Moreover, an efficient linearization technique is applied. By this technique, small cases can be solved exactly. However, as the size of the problem increases, the problem becomes computationally intractable and commercial solver requires substantial computation time and even finding the optimum solution for large-sized cases may not be possible. Therefore, the Iterated Local Search (ILS) heuristic algorithm is developed to find a near optimal solution based on geographical closeness.

The remainder of this paper is organized as follows. Section 2 reviews the literature relating to hub and spoke networks as well as the idea of reliability in them. Section 3 describes the proposed mathematical formulation for the problem in linear form. Section 4 presents the computational experiments. Finally, in Section 5, the conclusions and future work are presented.

\section{Literature Review}

In classical hub location problems, network designers try to find suitable hub locations from all the node locations and then proper allocation of non-hub nodes to minimize the total transportation costs. Extending this to real world scenarios often involves network designers considering multiple objectives [4] or uncertain parameters $[5,6,7]$ and network incompleteness $[8,9]$. In addition, the disruptive effects of natural disasters play an important role in determining the overall network performance. In the hub location literature, some researchers have considered the effect of disruption on hub location problems. We classify these studies into three categories: arc disruption; intentional disruption or interdiction; and hub disruption. Important studies from each category are presented below with a brief explanation.

\subsection{Arc disruption}

Initially, Kim and O'Kelly [10] studied the failure effect on hub networks. They considered reliability for each route. Reliability is evaluated by multiplying the probability of successful communication of each arc. The model tries to find hub locations and their allocations with maximum reliability. The authors applied this idea to both single and multiple allocation hub location problems. Moreover, they applied a mandatory separation distance between every pair of hubs. This constraint avoids hubs that are no closer together than a predefined distance. As a result, it avoids centralizing strategic facilities in one area of a network [11]. Davari et al. examined their single 
allocation model in a fuzzy environment, and they proposed a simulated annealing algorithm for solving the problem [12]. Kim [13] proposed a protective hub model. In this model, when a flow path is disrupted, it can be routed through another one-stop path. This path consists of an intermediate backup hub. The distance restrictions on backup hub links were also considered. As a result of this constraint, backup hubs could not be more than a predefined distance from each node.

\subsection{Intentional disruption or interdiction}

In the intentional disruption or interdiction model, a potential interdictor or attacker hits unprotected facilities so the attack creates maximal deficiency in the network [14]. Network designers try to minimize worst-case attacker hit effects. Recently, Parvaresh et al. modeled intentional disruption in a multiple allocation hub location problem using a multi-objective bi-level optimization approach [15]. In this study, the network designer and interdictor were considered as a leader and a follower, respectively. The leader's objectives are to minimize network total transportation costs under normal conditions and transportation costs after an $r$-interdiction ( $r$-interdiction means $r$ facilities from $p$ unprotected facilities are disrupted by an attacker), which should be minimized. The follower's objective is to maximize the total transportation cost of the leader's network after the disruption of $r$ facilities. They proposed a simulated annealing and tabu search to extract non-dominated solutions.

In another study, Lei proposed a new $r$-interdiction hub model [16]. In this model, $r$ hubs from $p$ established hubs might fail to operate due to natural causes or intentional disruption. The model tries to find the $r$ critical hubs such that in their disruption condition, the network will be faced with maximum transportation costs while each flow follows the lowest-cost path. This author also proposed a bi-level model where the service provider (leader) and users (followers) are decision makers. In this model, the leader attempts to find target critical hubs with maximum disruption effects in the network, while the users are seeking to minimize their transportation cost individually. A bi-level model that is similar to previous research and tried to minimize the worst-case scenario is therefore contributed. Recently, efficient solution approaches such as Benders decomposition[17], enumeration[18] and metaheuristic algorithms[19] have been developed to solve interdiction models. 


\subsection{Hub disruption}

An et al. investigated hub failure in both single and multiple allocations [20]. Their study suggested that the hub allocation structure should be changed by using backup hubs under disruption conditions. The objective function of their model minimizes normal transportation costs, transportation costs in disruptive situations, and costs of lost flows. They considered a single disruption in their model, which means that a disruption could occur in one hub at most in a single path. They proposed quadratic and linearized formulations. Moreover, they proposed Lagrangian relaxation and branchand-bound solution approaches to find high-quality, near-optimal or optimal solutions. In their proposed model, after the disruption and failure of a hub, nodes can be served by multiple backup hubs. This assumption is not realistic in single allocation structure networks like telecommunication problems; more discussion of this and the properties of such networks has been provided in $\operatorname{Kim}[11]$.

Azizi et al. proposed a mathematical model for the hub unavailability issue [21]. In their research, a hub may have failed after disruption, a backup hub supports it and handles disrupted hub operations to serve all its allocated demands. Mohammadi et al. proposed a reliable single allocation model with different allocation levels [22]. In their proposed nonlinear model, disruption occurs in hub facilities and the structure of hub facilities follow a tree structure. They also considered uncertain parameters in their proposed model. A recent research in this category is proposed in Mohammadi et al. [23]. Two objectives were considered in their model: median and center objectives. In the first one, total cost is minimized and in the second one, the network longest delivery time is minimized. The delivery time in hub facilities depends on the traveling time and hub operation time as a function of hub disruption rate.

Azizi developed a mathematical model to design single allocation hub and spoke networks under disruption of only one hub at any given time [24]. He developed three versions of particle swarm optimization algorithm to solve large-sized instances.

The scenario based models to consider the disruption in multiple and single allocation hub networks were presented by Yahyaei and Bashiri [25], Zhalechian et al. [26] and Rostami et al. [27] .

Most of the reviewed implicit models did not take into consideration massive disruption in the network where both hub facilities are subject to failure and non of them maintains singularity of assignment in case of disruption. 
On the other hand scenario based modeling in reliable facility location problem is that number of variables increase rapidly with increasing of the number of scenarios [28] and consequently significant computational time is required to solve the problem.

\subsection{This Work}

In the present study, we propose a lexicographic bi-objective model for the single allocation hub network, where each candidate node for establishing a hub facility has a predefined failure probability; massive disruptions can occur and both hubs in a path can be disrupted simultaneously, while the singularity of backup links is considered. Moreover, in this study, disrupted hubs are treated as normal nodes and backup hubs needed to serve them. In classical hub location problems, all nodes are served, and we apply the same strategy under both normal and disruption conditions. In addition, a heuristic algorithm based on iterated local search (ILS) [29] is proposed to solve the problem for large instances.

\section{The Hub Problem and the Reliable Version of it}

O'Kelly [30] proposed the single allocation hub location problem as the first model in the hub location literature. Generally, a hub facility is established in one of the existing nodes of the network and a non-hub node is connected to one of the established hubs. As seen in Fig. 1, consolidation effects occur in hub facilities (demonstrated between parts $a$ and $b$ ), and this leads to reductions in the cost of traveling through inter hub connections.

In this paper, the reliable singe allocation hub network design problem, that is independent of failure for each candidate hub location, is considered. The candidate nodes are a subset of demand nodes. The network is assumed to be a $p$-hub-Median problem with complete structure. A candidate point is called a reliable one if its failure probability is zero. An established hub among such points is therefore called a reliable hub. In the proposed reliable model, in addition to a normal path for a flow, the model tries to design a backup path for each flow (if necessary), and two allocations at most are possible for each demand node under normal and disruption conditions. Under normal conditions, node assignments follow the classical hub structure and each non-hub node is allocated to only one hub (hereafter called a primal $h u b)$. In case of disruption, nodes will be allocated to their backups. In 

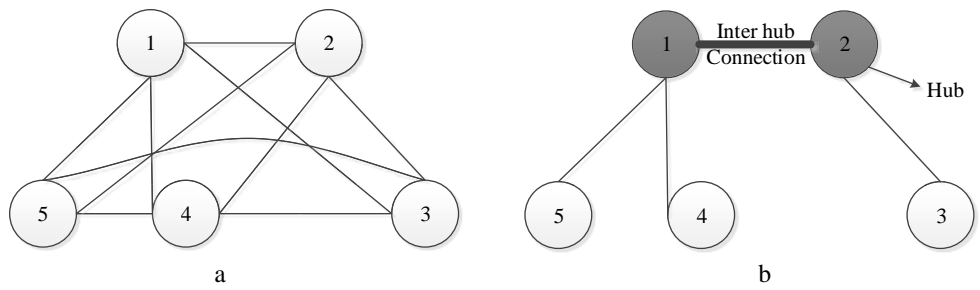

Figure 1: An origin-destination connections network b) A network based on hub facilities

this study, we assumed that only one backup allocation is allowable for each node. Since a hub is located in a demand node, it serves that node demand in normal conditions and after a hub facility disruption, the node should be allocated to its corresponding backup hub. A massive disruption can also occur in a network. This means that more than one hub may fail in a route, and the network will as well. By considering a disruptive environment, six conditions could occur to a hub network, which are depicted in Fig. 2.

In parts $a$ to $d$ of Fig. 2, backup hubs help the network to route flows, but in parts $e$ and $f$ some flows cannot be routed in the network while two hubs are disrupted. When both hubs in a route fail, the flow cannot be routed; we call this a lost flow. The lost flows can be divided into two categories. In the first category, suppose that both primal hubs of flow $i-j$ are disrupted when one or both of the failed hubs have been selected as backup hubs (see Fig. 2 $e$ as an example). It is clear that flow $i-j$ cannot be routed, and this failure is based on the network structure. We call this a Type I lost flow. In the second category, backup hubs are different from primal hubs, and lost flow occurs when primal and backup hubs fail simultaneously due to a massive disruption (see Fig. $2 f$ as an example). We call this a Type II lost flow. In the proposed model, we aim to decrease the total number of Type I and Type II lost flows in order to enhance network efficiency and responsiveness. The number of Type I lost flows is dependent on how nodes are allocated to the hubs and it can be controlled by adopting an appropriate allocation strategy.

Our model objectives are a reduction of Type I lost flows and minimizing 


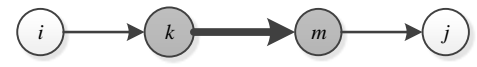

$\mathrm{a}$

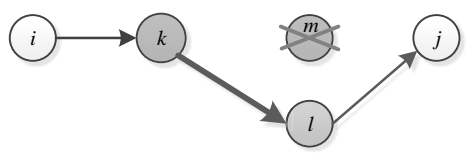

C

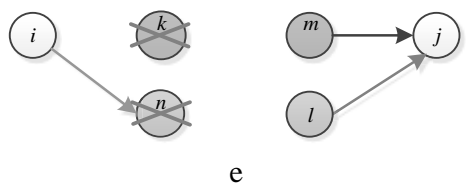

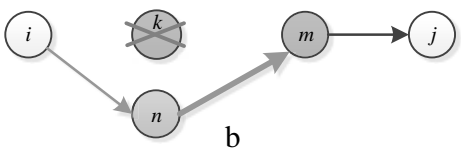

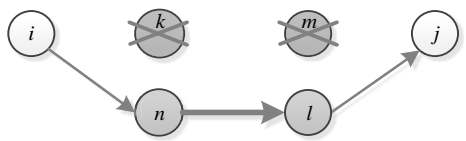

d

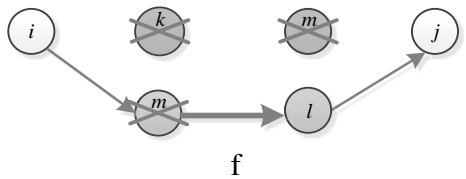

Figure 2: Different structures of a reliable hub network ( $i$ and $j$ are O-D while the green nodes are primal hub nodes and blue nodes are backup hubs). a) When there is no disruption in a path; b) Shows the disruption of the first hub and the alternative backup hub for node $i$; c) Shows disruption of the second hub; d) When both hubs in a route are disrupted and the flows are routed to backup hubs; e) Shows a Type I lost flow; f) Shows a Type II lost flow 
total transportation cost and lost flow costs under both normal and disruption conditions.

The mathematical model is given in Equations 1 - 25, This is denoted as Model 1.

Model $1: \operatorname{lex}\left(\min Z_{1}, \min Z_{2}\right)$

$Z_{1}=\sum_{i} \sum_{j} l_{i j}$

Expected total transportation cost under normal conditions:

$Z_{2}=\sum_{i} \sum_{j} \sum_{k} \sum_{m} w_{i j} C_{i j k m}\left(1-q_{k}-q_{m k}^{1}+q_{k} q_{m k}^{1}\right) x_{i j k m}$

Expected total transportation cost under disruption of all hubs:

$+\sum_{i} \sum_{j} \sum_{k} \sum_{m} \sum_{n} \sum_{l} w_{i j} C_{i j n l}\left(q_{k} q_{m k}^{2}\left(1-q_{n}\right)\left(1-q_{l n}^{1}\right)\right) x_{i j k m} t_{i j n l}$

Expected total transportation cost under disruption of the first hub:

$+\sum_{i} \sum_{j} \sum_{k} \sum_{m \neq k} \sum_{n} w_{i j} C_{i j n m}\left(q_{k}\left(1-q_{m}\right)\left(1-q_{n m}^{1}\right)\right) x_{i j k m} z_{i n}$

Expected total transportation cost under disruption of the second hub:

$+\sum_{i} \sum_{j} \sum_{k} \sum_{m \neq k} \sum_{n} w_{i j} C_{i j k n}\left(q_{m}\left(1-q_{k}\right)\left(1-q_{n k}^{1}\right)\right) x_{i j k m} z_{j n}$

Expected cost of unmet flows under disruption of both hubs:

$+\sum_{i} \sum_{j} \sum_{k} \sum_{m} \sum_{n} \sum_{n} \sum_{l} w_{i j} L F_{i j}\left(q_{k} q_{m k}^{2}\left(1-\left(1-q_{n}\right)\left(1-q_{l n}^{1}\right)\right)\right) x_{i j k m} t_{i j n l}$ 
Expected cost of unmet flows under disruption of the first hub:

$+\sum_{i} \sum_{j} \sum_{k} \sum_{m \neq k} \sum_{n} w_{i j} L F_{i j}\left(q_{k}\left(1-q_{m}\right) q_{n m}^{1}\right) x_{i j k m} z_{i n}$

Expected cost of unmet flows under disruption of the second hub:

$+\sum_{i} \sum_{j} \sum_{k} \sum_{m \neq k} \sum_{n} w_{i j} L F_{i j}\left(q_{m}\left(1-q_{k}\right) q_{n k}^{1}\right) x_{i j k m} z_{j n}$

Expected cost of Type I lost flows:

$+\sum_{i} \sum_{j} \sum_{k} \sum_{m} w_{i j} L F_{i j}\left(q_{k} q_{m k}^{2}\right) x_{i j k m} l_{i j}$

s.t: $\sum_{m} x_{i j k m}=y_{i k} \forall i, j, k$

$\sum_{k} x_{i j k m}=y_{j m} \forall i, j, m$

$y_{i k} \leq y_{k k} \forall i, k$

$\sum_{k} y_{i k}=1 \forall i$

$\sum_{k} y_{k k}=p$

$z 1_{i} \leq y_{i i} \forall i$

$z_{i k} \leq z 1_{k} \forall i, k$ 


$$
\begin{aligned}
& z_{i k}+y_{i k} \leq 1+R_{k} \forall i, k \\
& y_{i k}+\sum_{j \neq k} z_{i j} \leq 2-R_{k} \forall i, k \\
& \sum_{m} s_{i j k m}=z_{i k} \forall i, j, k \\
& \sum_{k} s_{i j k m}=z_{j m} \forall i, j, m \\
& \sum_{k} z_{i k}=1 \forall i
\end{aligned}
$$

$s_{i j k m}+\sum_{l} x_{i j m l}+\sum_{l \neq m} x_{i j l k}-l_{i j}=1+R_{k} \cdot R_{m} \forall i, j, k, m$

$t_{i j k m} \geq s_{i j k m}-l_{i j} \forall i, j, k, m$

$0 \leq s_{i j k m}, x_{i j k m} \forall i, j, k, m$ and the other variables are binary 


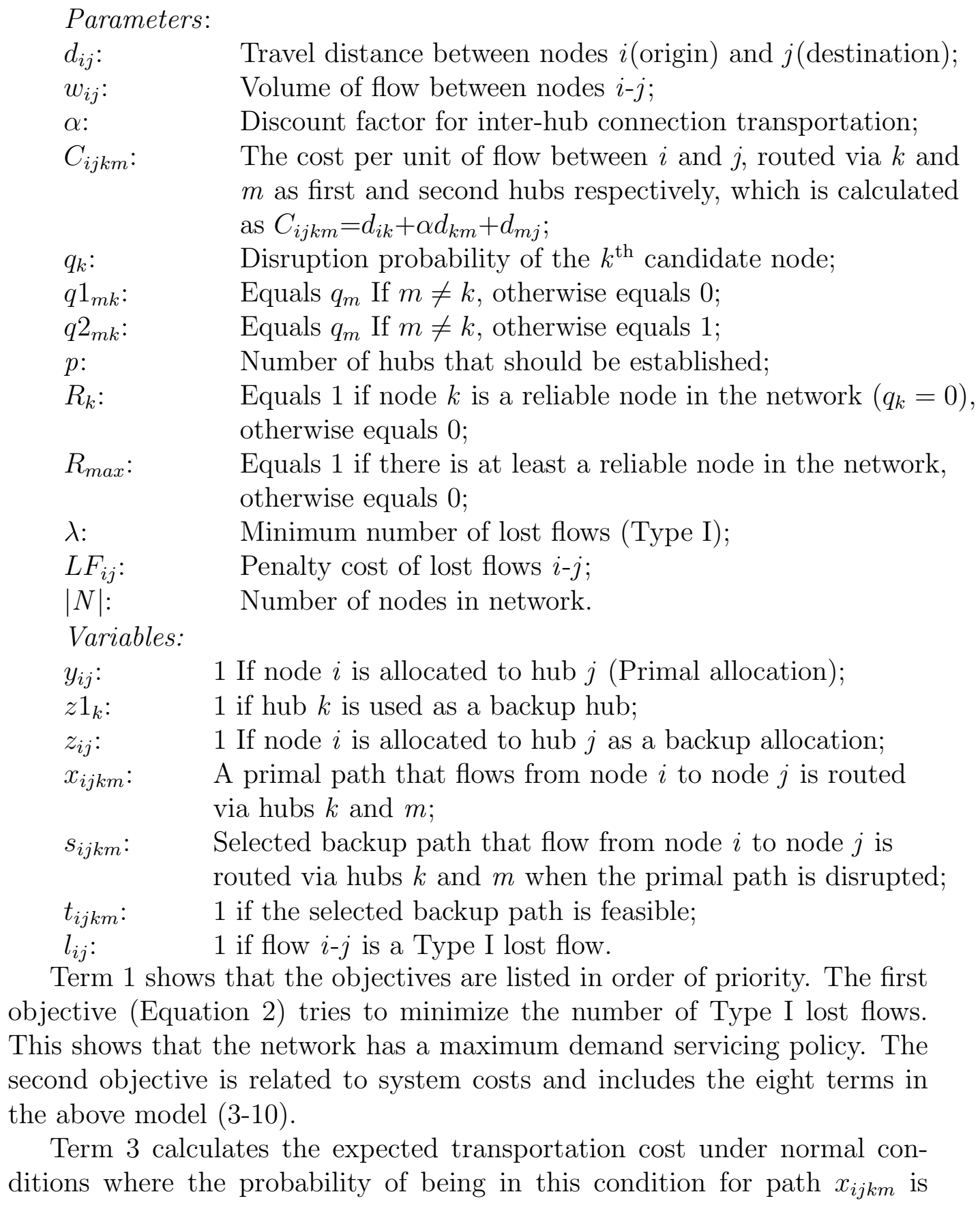

Term 1 shows that the objectives are listed in order of priority. The first objective (Equation 2) tries to minimize the number of Type I lost flows. This shows that the network has a maximum demand servicing policy. The second objective is related to system costs and includes the eight terms in the above model (3-10).

Term 3 calculates the expected transportation cost under normal conditions where the probability of being in this condition for path $x_{i j k m}$ is calculated as: 
$\left.\begin{array}{c}\text { One - Stop path } i \rightarrow k \rightarrow k \rightarrow j 1-q_{k} \\ \text { Two-Stop path } i \rightarrow k \rightarrow m \rightarrow j 1-q_{k}-q_{m}+q_{k} q_{m}\end{array}\right\} 1-q_{k}-q_{m k}^{1}+q_{k} q_{m k}^{1}$

Term 4 shows the total expected transportation cost when the intermediate hub in a one-stop path or both intermediate hubs in a two-stop path are disrupted and none of backup hub(s) are disrupted. For each path, the probability of this status is equal to $q_{k} q_{m k}^{2}$.

Term 5 shows the total expected transportation cost when only the first hub is disrupted in a two-stop path and the backup hub for the first hub is working. Term 6 shows total expected transportation cost when only the second hub is disrupted in a two-stop path and the backup hub for the first hub is working. Terms 7-10 calculate total expected cost of lost flows.

Constraints 11-15 ensure consistency of the network with the classical hub network primary constraints. Constraint 16 guarantees that the backup hubs will be selected from the primal hub set as well. Under constraint 17, all nodes are assigned to hubs opened as backup hubs. Constraint 18 ensures node $i$ cannot have a primal and backup link to hub $k$ simultaneously, unless hub $k$ is a reliable hub. Constraint 19 ensures that nodes allocated to a reliable hub cannot be allocated to another backup hub. Constraints 20 and 21 ensure that flow $i-j$ cannot be routed to a backup route unless backup hubs are related to that route have been allocated to $i-j$ before. Constraint 22 guarantees a single allocation strategy in a disruption situation. Consider that nodes $i$ and $j$ are assigned to primal hubs $k$ and $m$ respectively. When both hubs in this route are disrupted, $k$ and $m$ cannot be used as backups for nodes $i$ and $j$, respectively. Constraint 23 tries to enforce this condition. However, as we show later, this condition is not always possible for some flows, and in such cases (Type I lost flows), the selected backup route should be considered a lost flow and cannot be routed physically. Constraint 24 extracts the feasible routes from the selected backup routes. Flow between nodes $i$ and $j$ is considered a lost flow; hence, the objective function tries to minimize with the feasible route being zero.

\subsection{Transforming a lexicographic bi-objective model into a single objective model}

In the single allocation hub location problem, each node is restricted to be served by a hub, and all the flows are served. As it is mentioned, we considered only one primal and one backup hub for each node. However, 
in some circumstances, part of flows in the network may not be feasible (unserved). In this paper, we considered a maximum servicing policy, and the minimization of the total number of lost flows (unserved flows) is considered as objective while this objective has priority over a network cost. In classical model servicing all the flows are considered as constraints. However, a similar constraint is not valid here for all cases of disruption.

In order to attain optimal solution of Model 1, a two-step optimization approach is needed. In Step 1, the first objective is optimized; in Step 2, the second objective is optimized while a constraint that guarantees optimality of the first objective is added to the model. In this section, we transform the lexicographic bi-objective model into a single objective model. Had there not been a definite priority between objective functions 1 and 3, a multiobjective approach could have been used. By this transformation, we can solve the model in a single step instead of two steps.

Assume that the first objective optimal value is $\lambda$ (Minimum number of Type I lost flows). Moreover, assume that there is no reliable point and as a result, there is no reliable hub on the network. For a better illustration, assume that the network assignments are similar to Fig. 3. Non-hub nodes $i, j$ and $i^{\prime}$ are assigned to primal hubs $k, m$ and $n$ respectively. Hubs $n$ and $l$ are chosen as backups for nodes $i$ and $j$ respectively. This means that $x_{i j k m}$ and $s_{i j n l}$ are equal to 1 . According to the network depicted in Fig. $3, s_{i j n l}$ is a feasible selected path. Now consider $x_{i i^{\prime} k n}$, a primal path between nodes $i$ and $i^{\prime}$. When both hubs are disrupted in this path, the selected backup path is $s_{i i^{\prime} n b}$, where $b$ is one of nodes $k, m$ or $l$ and it is infeasible because this path contains hub $n$, which is disrupted. $s_{i n n b^{\prime}}$ is also an infeasible path where $b^{\prime}$ is the backup for disrupted hub $n$ and is one of nodes $k, m$ or $l$. In the following, we attempt to find the minimum number of Type I lost flows for an arbitrary network (like Fig. 3).

Definition 1: A hub is a semi-isolated hub if the only node assigned to this hub is itself in primal allocations.

Theorem 1: Suppose that there is no reliable point. In this case, the network has the least number of Type I lost flows for non-hub nodes if all assigned backup hubs for non-hub nodes are semi-isolated hubs.

Proof. It is obvious that for origin non-hub node $i$ assigned to backup hub $k$ these backup paths are not feasible: $s_{i j k x}, x \in\{H \backslash k\}, j \in a$; where $H$ is the set of hubs and $a$ is the set of the nodes assigned to hub $k$ in a primal allocation and $1 \leq|a| \leq|N|-p-1$. Therefore, the range of Type I lost 


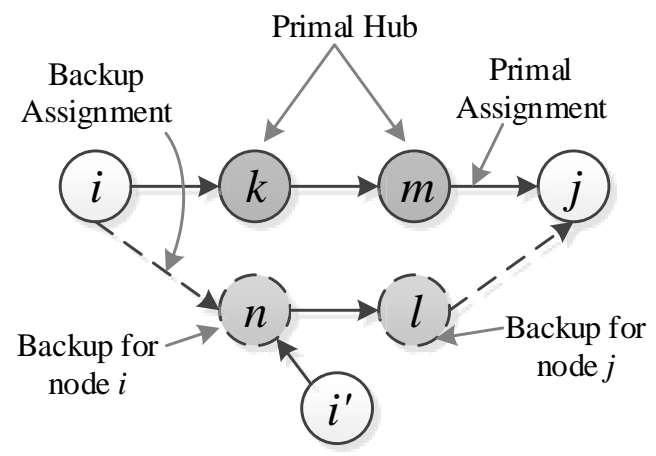

Figure 3: An illustrative example for different assignments in a hub network with backups

flows for each origin node varies from 1 to $|N|-p-1$. If they are allocated to semi-isolated hubs by backup assignment, they will have the least Type I lost flows; and conversely, if node $i$ is considered as the destination, this deduction is valid.

Corollary 1: Whenever there is no possibility of establishing a reliable hub, the lowest total number of Type I lost flows for non-hub nodes is $2 \times$ $(|N|-p)$.

Proof. Due to the previous theorem, if each node such as $i$ is assigned to a semi-isolated hub such as $k$, flow origins from $i$ to destination $k$ are infeasible; moreover, the same flow origins from $k$ to destination $i$ are infeasible as well. Since there are $|N|-p$ non-hub nodes, we have $2 \times(|N|-p)$ Type I lost flows and the corollary is proved.

Definition 2: Hubs $i$ and $j$ are teammate hubs if hub $i$ is used as backup for $j$ and hub $j$ is used as backup for $i$.

Theorem 2: Assume that there is no reliable point in the network. In this condition, the network has the lowest number of Type I lost flows for demand of hub nodes if it has the maximum possible teammate hubs.

Proof. First, assume that $p$ is even. We can demonstrate a graph equivalent to hub nodes and their backup assignments (see Fig. 4). As is clear, each edge $(e)$ in the demonstrated graph shows two lost flows (two backup assignments in a teammate hub are demonstrated by one edge). For example, $e_{l m}$ shows that $s_{l m m k}$ and $s_{m l k m}$ are infeasible (flows from $l$ to $m$ and from $m$ to $l$ are infeasible when both hubs are disrupted). On the other hand, since all nodes should have backup hub assignments, each node degree is equal to or greater than 1 in the graph $\left(d_{i} \geq 1\right)$. If we have the maximum 


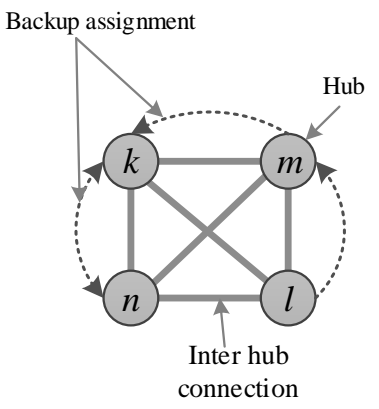

a

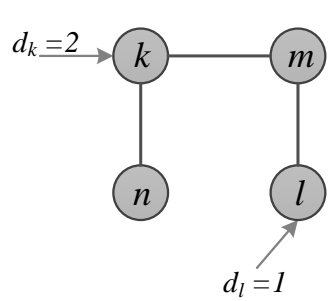

$\mathrm{b}$

Figure 4: Inter-hub connection, backup assignment and equivalent backup assignment graph

number of teammate hubs in a network, the lowest value for each node degree is achieved $\left(d_{i}=1 \forall i\right)$. A similar conclusion is valid when $p$ is odd, but it should be noted that we have $d_{i}=2$ for one hub node like $i$.

Corollary 2: In a network without reliable points, the lowest number of Type I lost flows for hub nodes is $p$ if $p$ is even and $p+1$ if $p$ is odd.

According to the previous theorem, if $p$ is even, we have $d_{i}=1 \forall i$. We have $|E|=\frac{1}{2} \sum_{i} d_{i}=p$ where $|E|$ is the number of edges in the equivalent backup assignment graph. As mentioned, for each edge in the graph we have two Type I lost flows and the value is equivalent to for a hub node. If $p$ is odd, the number of Type I lost flows is $\sum_{i} d_{i}=p+1$.

By corollary 1 and 2, we can calculate the lowest number of Type I lost flows (the optimal value of the first objective) using the following equation:

$\lambda=2 \times(|N|-p)+p+\beta=2|N|-p+\beta$

where $\beta=(p \bmod 2)$. It is worth noting that if there is a reliable hub in the network, a solution for the model is a network in which the reliable hub is used as backup for all flows. Therefore, we can have no lost flows (Type I and II) in the network. Now, by adding the following constraint to the model, we can omit the first objective and change the bi-objective model into a single 
objective model.

$\sum_{i} \sum_{j} l_{i j}=2 \times \lambda\left(1-\max _{k}\left\{R_{k}\right\}\right)$

\subsection{Linearization}

Many techniques have been proposed for linearization of zero-one quadratic problems. In order to transform our quadratic model into a linear model, we applied an efficient linearization technique that is proposed in He et al. [31]. Suppose that there is a quadratic model similar to the following:

$$
\begin{gathered}
\min \sum_{i \in N} \sum_{j \in M} d_{i j} x_{i} t j \\
\text { s.t }: A_{i} x_{i}+B_{j} t_{j} \leq C \\
x_{i}, t_{j} \in\{0,1\} \forall i, j
\end{gathered}
$$

where $d_{i j} \geq 0$. It can be proved that the above model is equivalent to the following model:

$$
\begin{gathered}
\min \sum_{i \in N}\left(s_{i}-\sigma x_{i}\right) \\
\text { s.t }: A_{i} x_{i}+B_{j} t_{j} \leq C \\
\sum_{j \in M} d_{i j} t_{j}+\sigma=s_{i}+y_{i} \forall i \in N \\
y_{i} \leq(L+\sigma)\left(1-x_{i}\right) \forall i \in N \\
x_{i}, t_{j} \in\{0,1\}, y_{i}, s_{i} \geq 0 \forall i, j
\end{gathered}
$$

where the relaxation parameter $(\sigma)$ is equal to or greater than 0 and

$$
L=\max _{i \in N} \sum_{j \in M} d_{i j}
$$

After this presentation, we propose a linear form of Model 1:

Theorem 3: The formulations in Model 1 are equivalent to the following minimization problem: 
Model 2:

$$
\begin{aligned}
\min Z= & \sum_{i} \sum_{j} \sum_{k} \sum_{m} w_{i j} C_{i j k m}\left(1-q_{k}-q_{m k}^{1}+q_{k} q_{m k}^{1}\right) x_{i j k m} \\
& +\sum_{i} \sum_{j} \sum_{k} \sum_{m}\left(S 1_{i j k m}-\sigma t_{i j k m}\right)+\sum_{i} \sum_{j}\left(S 2_{i j}-\sigma z_{i j}\right) \\
& +\sum_{i} \sum_{j}\left(S 3_{i j}-\sigma z_{i j}\right)+\sum_{i} \sum_{j}\left(S 4_{i j}-\sigma l_{i j}\right)
\end{aligned}
$$

$$
\begin{aligned}
& \sum_{k} \sum_{m} w_{i j}\left(C_{i j n l}\left(q_{k} q_{m k}^{2}\left(1-q_{n}\right)\left(1-q_{l n}^{1}\right)\right)+L F_{i j}\left(q_{k} q_{m k}^{2}\left(1-\left(1-q_{n}\right)\left(1-q_{l n}^{1}\right)\right)\right)\right) x_{i j k m} \\
& +\sigma=S 1_{i j n l}+Y 1_{i j n l} \forall i, j, n, l
\end{aligned}
$$

$$
\begin{aligned}
& Y 1_{i j n l} \leq(L 1+\sigma)\left(1-t_{i j n l}\right) \forall i, j, n, l \\
& \sum_{j} \sum_{k} \sum_{m \neq k} w_{i j}\left(C_{i j n m}\left(q_{k}\left(1-q_{m}\right)\left(1-q_{n m}^{1}\right)\right)+L F_{i j}\left(q_{k}\left(1-q_{m}\right) q_{n m}^{1}\right)\right) x_{i j k m} \\
& \quad+\sigma=S 2_{i n}+Y 2_{i n} \forall i, n
\end{aligned}
$$

$$
\begin{aligned}
& Y 2_{i n} \leq(L 2+\sigma)\left(1-z_{i n}\right) \forall i, n \\
& \quad \sum_{j} \sum_{k} \sum_{m \neq k} w_{i j}\left(C_{i j k n}\left(q_{m}\left(1-q_{k}\right)\left(1-q_{n k}^{1}\right)\right)+L F_{i j}\left(q_{m}\left(1-q_{k}\right) q_{n k}^{1}\right)\right) x_{i j k m} \\
& \quad+\sigma=S 3_{i n}+Y 3_{i n} \forall i, n
\end{aligned}
$$

$$
Y 3_{i n} \leq(L 3+\sigma)\left(1-z_{i n}\right) \forall i, n
$$




$$
\begin{aligned}
& \sum_{k} \sum_{m} w_{i j} L F_{i j}\left(q_{k} q_{m k}^{2}\right) x_{i j k m}+\sigma=S 4_{i j}+Y 4_{i j} \forall i, j \\
& Y 4_{i j} \leq(L 4+\sigma)\left(1-l_{i j}\right) \forall i, j \\
& S 1_{i j k m} \text { and } Y 1_{i j k m} \geq 0 \forall i, j, k, m ; S 2_{i j}, S 3_{i j}, S 4_{i j}, Y 2_{i j}, Y 3_{i j}, Y 4_{i j} \geq 0 \forall i, j
\end{aligned}
$$

where $L 1, L 2, L 3$ and $L 4$ can be calculated according to Equation 28; the calculation will be discussed presently.

Proof. Necessity: Since $y_{i k} \forall i, k$ is binary and according to Equations 11, 12 and 14, $x_{i j k m}$ has binary behavior. Similar to the proof in He et al. [32], assume $B^{*}$ is the optimal solution of Model 2. By multiplying $t_{i j n l}^{*}$ on both sides of the Equations 30-38 and summing them over all $i, j, n$ and $l$ we get:

$$
\begin{aligned}
& \sum_{i} \sum_{j} \sum_{k} \sum_{m} \sum_{n} \sum_{l} w_{i j}\left(C_{i j n l}\left(q_{k} q_{m}^{2} k\right)\left(1-q_{n}\right)\left(1-q_{l}^{1} n\right)\right) \\
& +L F_{i j}\left(q_{k} q_{m k}^{2}\left(1-\left(1-q_{n}\right)\left(1-q_{l n}^{1}\right)\right)\right) x_{i j k m} t_{i j n l}^{*} \\
& +\sum_{i} \sum_{j} \sum_{n} \sum_{l} \sigma t_{i j n l}^{*}=\sum_{i} \sum_{j} \sum_{n} \sum_{l}\left(S 1_{i j n l}+Y 1_{i j n l}\right) t_{i j n l}^{*}
\end{aligned}
$$

Moreover, by 31 and $Y 1_{i j n l} \geq 0$; we have:

$$
Y 1_{i j n l}^{*} t_{i j n l}^{*}=0 \quad \forall i, j, n, l
$$

According to Equations 39 and 40, proof of the following equation leads to proof of equivalence of the objective functions for the two models:

$$
S 1_{i j n l}^{*} t_{i j n l}^{*}=S 1_{i j n l}^{*} \forall i, j, n, l
$$

This is equivalent to the proof that for any $i, j, n$ and $l$ if $t_{i j n l}^{*}=0$ then $S 1_{i j n l}^{*}=0$. Reasoning by contradiction, let us assume that for certain $i^{\prime}, j^{\prime}, n^{\prime}$ and $l^{\prime}$ of equation 41 are not valid; this means that $t_{i^{\prime} j^{\prime} n^{\prime} l^{\prime}}^{*}$ and $S 1_{i^{\prime} j^{\prime} n^{\prime} l^{\prime}}^{*}>0$. 
To exhibit this contradiction; we created a solution like $B^{\prime}$ where $Y 1_{i^{\prime} j^{\prime} n^{\prime} l^{\prime}}^{\prime}=$ $Y 1_{i^{\prime} j^{\prime} n^{\prime} l^{\prime}}^{*}+S 1_{i^{\prime} j^{\prime} n^{\prime} l^{\prime}}^{*}, S 1_{i^{\prime} j^{\prime} n^{\prime} l^{\prime}}^{\prime}$ and the values of the other variables are the same as $B^{*}$. It can be confirmed that $B^{\prime}$ is a feasible solution for Model 2 with a lower objective function value. Thus, this result contradicts the assumption that $B^{*}$ is the optimal solution. This proof is valid for other quadratic terms.

Sufficiency: The proof is similar.

Equation 28 proposes very loose bounds on $Y 1, Y 2, Y 3$ and $Y 4$. Therefore, we strengthened these bounds based on the model structure. According to Equation 28, the bound for $Y 1$ is $\sum_{k} \sum_{m} w_{i j} C_{i j n l}\left(q_{k} q_{m k}^{2}\left(1-q_{n}\right)\left(1-q_{l n}^{1}\right)\right)+$ $L F_{i j}\left(q_{k} q_{m k}^{2}\left(1-\left(1-q_{n}\right)\left(1-q_{l n}^{1}\right)\right)\right) \forall i, j, n, l$. However, the model follows the classical single allocation hub problem; for each $i$ and $j$ we have:

$$
\sum_{k} \sum_{m} x_{i j k m}=1
$$

This means that we have:

$$
\begin{aligned}
& \sum_{k} \sum_{m} w_{i j}\left(C_{i j n l}\left(q_{k} q_{m k}^{2}\left(1-q_{n}\right)\left(1-q_{l n}^{1}\right)\right)+L F_{i j}\left(q_{k} q_{m k}^{2}\left(1-\left(1-q_{n}\right)\left(1-q_{l n}^{1}\right)\right)\right)\right) x_{i j k m} \\
& \leq \overbrace{\max _{k, m} w_{i j}\left(C_{i j n l}\left(q_{k} q_{m k}^{2}\left(1-q_{n}\right)\left(1-q_{l n}^{1}\right)\right)+L F_{i j}\left(q_{k} q_{m k}^{2}\left(1-\left(1-q_{n}\right)\left(1-q_{l n}^{1}\right)\right)\right)\right)}^{L 1_{i j n l}} \forall i, j, n, l \\
& \leq \sum_{k} \sum_{m} w_{i j}\left(C_{i j n l}\left(q_{k} q_{m k}^{2}\left(1-q_{n}\right)\left(1-q_{l n}^{1}\right)\right)+L F_{i j}\left(q_{k} q_{m k}^{2}\left(1-\left(1-q_{n}\right)\left(1-q_{l n}^{1}\right)\right)\right)\right)
\end{aligned}
$$

Therefore, we can use the second term in the above inequality instead of third term. A similar conclusion is valid for $Y 4$. To obtain tighter upper bounds for $Y$ 2, for each $i$ we have:

$$
\sum_{j} \sum_{k} \sum_{m} x_{i j k m}=|N|
$$

So, the following tightened bounds can be used for Y2:

$$
L 2_{i n}=\sum_{j \in N} \max _{k, m \neq k} w_{i j}\left(C_{i j n m}\left(q_{k}\left(1-q_{m}\right)\left(1-q_{n m}^{1}\right)\right)+L F_{i j}\left(q_{k}\left(1-q_{m}\right) q_{n m}^{1}\right)\right) x_{i j k m} \forall i, n
$$

Tighter bounds for $Y 3$ can be created according to a similar process. 


\subsection{Iterated local search for a reliable single allocation hub location problem}

The linearization technique applied in the previous section can improve the computational ability of the model. However, due to the complexity of the model, this improvement may not be effective in large instances. From a computational complexity point of view, Kara proved that the single allocation $p$-hub problem is NP-Hard[33], therefore an efficient heuristic is needed to solve large instances of this problem. The reliable model is a generalization of single allocation $p$-hub, and an efficient heuristic is needed for solving this model as well.

The applied heuristic for treating NP-hard combinatorial optimization problem of this research is Iterated Local Search (ILS). The ILS is well known for its effectiveness and simplicity in practice [34] and it can escape from a local minimum without losing of the good attributes of the current solution. It has been successfully implemented for many optimization problems, such as the vehicle routing problem [35], inventory routing problems [36], university course timetabling [37] and machine scheduling [38].

The basic ILS algorithm contains four major steps: 1) generating an initial solution (S0), 2) generating solution by local search (SL), 3) perturbation solution (SP), and 4) termination criteria. The S0 can be generated randomly or a special heuristic can be used. In our implementation, a random procedure is used. Then, to improve the S0, new solutions (SL) are generated by a local search procedure. Next, one of SL is selected and perturbed (SP). The local search is applied again for SP. A new solution can be accepted according to the specified rule. This process is repeated until termination criteria are satisfied. The basic algorithm is developed for reliable hub location problem and is briefly described in following. We assume that there exists at least a reliable point within the network (a basically similar explanation can be mentioned if there is no reliable point within the network). In order to gain a better understanding, Fig. 5 illustrates on how the algorithm works. To start the main steps of ILS algorithm, a feasible solution in the problem should be encoded. As it is mentioned there are $|N|$ nodes and $p$ hubs should be selected. In Fig 5 (a), there are nine nodes within the network and we intend to establish three hubs. The red dashed line nodes are reliable ones (nodes 2, 4 and 5). Moreover, a primal and backup assignment should be considered for each node. According to the explanation in section 3.1, the optimal solution contains at least a reliable hub. Therefore, one of the $p$ hubs should be selected among the reliable nodes (Fig. 5 (b), nodes 4 and 5 are 


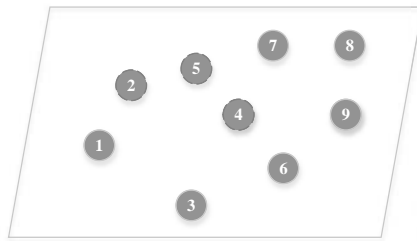

(a) Restricted Change

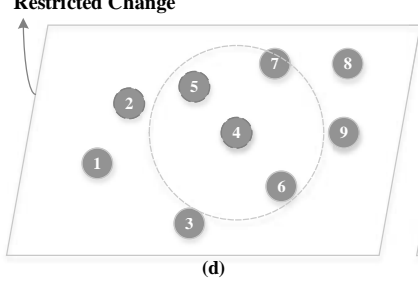

(d)

Correspond Solution Representation: 4-5-7|5-5-4-4-5-4-7-7-7 | 5-5-4-5-5-5-4-4
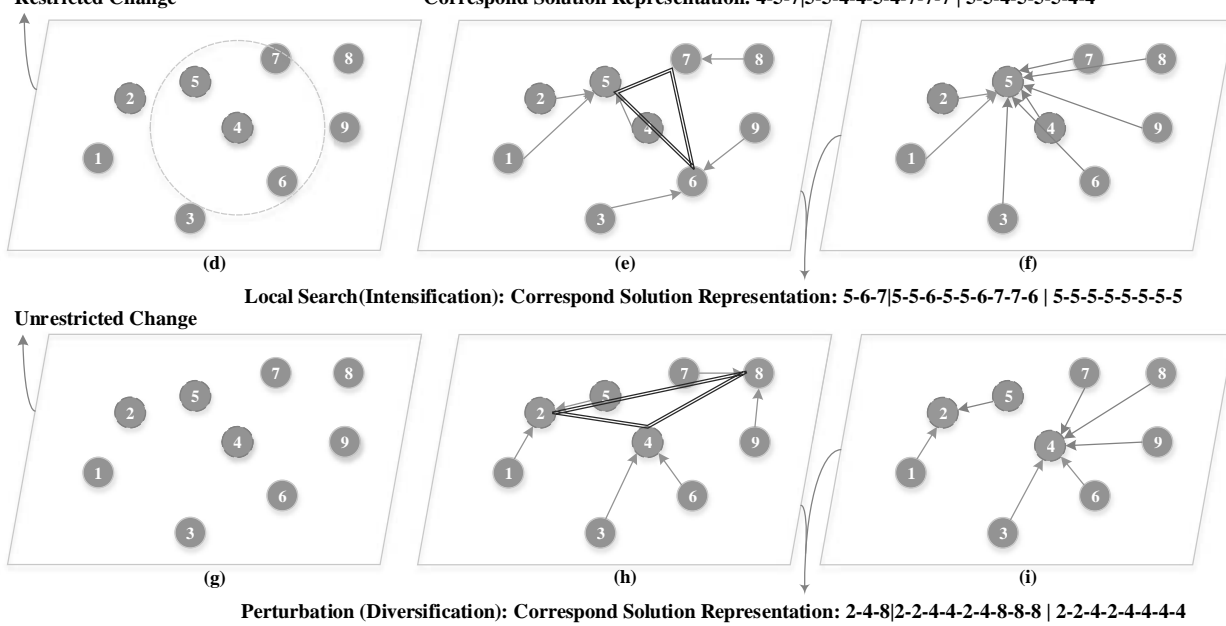

Figure 5: Graphical presentation of ILS for reliable single allocation hub network design 
selected). Primal and backup allocations of nodes are based on the nearest distance (Fig. 5 (c)), but for backup ones, a hub should be selected among the reliable hubs (Fig. 5 (c)). As depicted on Fig. 5, encoded solution of this feasible network contains three parts: (4-5-7) represent the established hubs, (5-5-4-4-5-4-7-7-7) represent primal assignments and lastly, (5-5-4-5-5-5-4-4) indicate backup assignments. In the global search approaches, intensification and diversification are two main operations which should be considered. By using diversification operation, different domains of solution are searched and by using intensification, a specified part of space is searched to find highquality solutions [39]. ILS contains two main procedures, local search and perturbation. In this paper, we aim to local search as intensifier operator and perturbation as a diversifier. In local search phase, the aim is to find a random similar solution. Clearly, the network structure is highly depended on the location of hubs. So to find a similar solution a small part of the selected hub should be changed, for example, one of the three hubs (node 4 should is chosen randomly in Fig. $5(\mathrm{~d})$ ). Moreover, the new hub should be selected within a specified distance (orange dashed line). Then, the primal and backup assignments are created according to previous explanation (Fig. 5 (e and $\mathrm{f})$ ). These steps are done repeatedly for specified times. It is worthy of noting that selecting of new solution is according to the always the best known solution [40]. In Perturbation phase, the aim is to explore new regions of solution space. Therefore, more hubs can be replaced by new ones and there is no distance restriction in selection of new hubs (Fig. 5 (g)). Fig. 5 (h) and (i) show the updated primal and the backup assignments, respectively. This procedure is repeated until stopping criterion (number of iterations) is satisfied. The proposed algorithm is summarized by the provided flowchart shown in Fig. 6.

\section{Computational analysis}

In this research, we considered two cases with 8 and 10 nodes of the CAB data set [30]. We applied CPLEX software for optimization. All of the computational tests were carried out on a laptop computer with an INTEL Core i5 CPU with $2.5 \mathrm{GHz}$ clock speed and $4 \mathrm{~GB}$ of RAM. Failure probability was randomly generated from $(0,0.1]$. Moreover, two and three random nodes from the data for 8 and 10 nodes are considered to be reliable nodes in the computations in Table 2. The value of $L F_{i j}$ is equal to $3 d_{i j}$ and $\sigma$ is equal to zero. For comparison of the linearization technique, three modes are consid- 


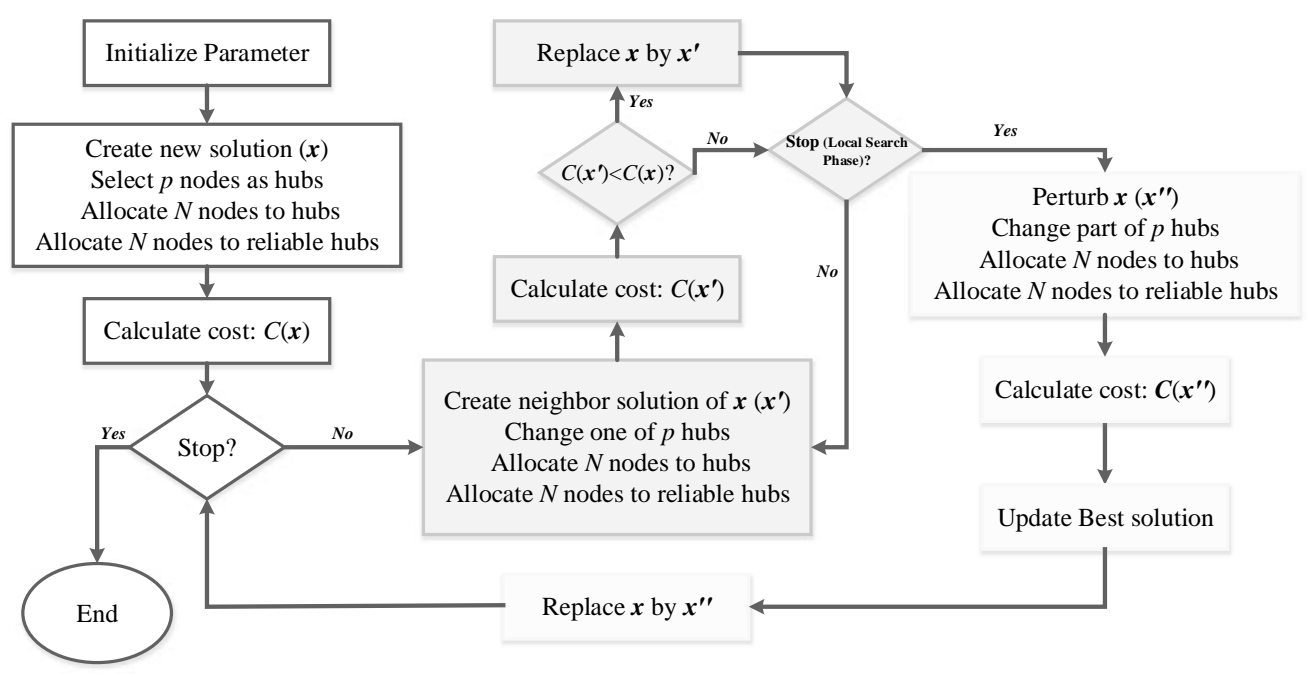

Figure 6: An illustrative flowchart of the proposed algorithm

ered: loose bounds (LB) calculated according to Equation 28, tight bounds (TB) as proposed in this research according to Equations 43 and 45, and the bilinear-programming-based linearization (BP) method proposed by Sherali and Smith [41].Their values were the same as reported for tight bounds. In the Test Problem column, the first number indicates the number of nodes in the network; the second number shows the number of hubs that should be located; and the third number shows the discount factor value. The values of OFV (Objective Function Value) and Location have been deleted for loose bounds and bilinear linearization in Tables 1 and 2 for simplicity, while their values are equal to the values of the tight bounds.

As is clear in Table 1 and Table 2, tight bounds work better in most test problems. In both tables, the objective values are equal in the three approaches while the CPU time in seconds (denoted by Time in Table 1 and Table 2) of the proposed approach is less than the other two approaches in most cases.

\subsection{Analysis of network performance}

Network performance is an important issue in the evaluation of network design. Network performance can be defined as the capability of serving 
Table 1: Optimization results for the selected data set without reliable points

\begin{tabular}{|l|c|c|c|c|c|}
\hline & \multicolumn{3}{|c|}{ Time(Sec) } & Locaion & OFV \\
\hline Test Problem & LB & TB & BP & & \\
\hline $10-3-0.8$ & 2493.5 & $\mathbf{1 7 4 8 . 3 7}$ & 3548.95 & $4-6-7$ & 733601861.4 \\
\hline $10-3-0.2$ & $\mathbf{1 0 0 9 . 8 7}$ & 1226.29 & 2351.96 & $4-6-7$ & 515278569.5 \\
\hline $10-2-0.8$ & 394.93 & 612.68 & $\mathbf{3 3 9 . 0 4}$ & $4-6$ & 926335484.6 \\
\hline $10-2-0.2$ & 888.21 & $\mathbf{3 5 2 . 2 3}$ & 566.07 & $6-7$ & 840004664.7 \\
\hline $8-3-0.8$ & 2761.57 & $\mathbf{1 4 3 . 0 9}$ & 2347.23 & $4-5-6$ & 490589221.4 \\
\hline $8-3-0.2$ & 165.8 & $\mathbf{1 3 9 . 3 4}$ & 215.37 & $4-6-7$ & 351267219 \\
\hline $8-2-0.8$ & $\mathbf{9 3 . 9 6}$ & 94.54 & 102.82 & $4-6$ & 545709836.1 \\
\hline $8-2-0.2$ & 88.8 & $\mathbf{7 1 . 2}$ & 100.09 & $3-4$ & 481765105.2 \\
\hline
\end{tabular}

Table 2: Optimization results for the selected data set with reliable points

\begin{tabular}{|l|c|c|c|c|c|}
\hline & \multicolumn{3}{|c|}{ Time(Sec) } & Locaion & OFV \\
\hline Test Problem & LB & TB & BP & & \\
\hline $10-3-0.8$ & 128.53 & $\mathbf{9 2 . 4 2}$ & 229.39 & $6-8-10$ & 794235690.2 \\
\hline $10-3-0.2$ & $\mathbf{1 3 6 . 2 1}$ & 136.73 & 214.76 & $2-4-7$ & 561106570.6 \\
\hline $10-2-0.8$ & 84.59 & $\mathbf{7 8 . 7 8}$ & 97.67 & $6-10$ & 865365787.8 \\
\hline $10-2-0.2$ & $\mathbf{7 6 . 3 9}$ & 82.09 & 91.26 & $6-10$ & 678373626.5 \\
\hline $8-3-0.8$ & 30.93 & $\mathbf{2 8 . 3 6}$ & 36.39 & $4-5-6$ & 464833580.8 \\
\hline $8-3-0.2$ & 31.09 & $\mathbf{2 9 . 5 6}$ & 35.25 & $2-4-7$ & 337337014.3 \\
\hline $8-2-0.8$ & $\mathbf{2 1 . 5 6}$ & 22.32 & 22.48 & $4-6$ & 495236399.1 \\
\hline $8-2-0.2$ & 23.37 & 23.84 & $\mathbf{2 2 . 8 2}$ & $6-7$ & 433383900.2 \\
\hline
\end{tabular}


various traffic demands placed on the network [42]. We can define an adapted meaning for network performance in disruption mode as the capability of the network to serve the demands in normal and disruption situations. By this definition, we can calculate classical and reliable hub network performance according to Equations 46 and 47:

Classic network performance measure:

$$
\left(\sum_{i} \sum_{j} \sum_{k} \sum_{m} w_{i j}\left(1-q_{k}-q_{m k}^{1}+q_{k} q_{m k}^{1}\right) x_{i j k m}\right) / \sum_{i} \sum_{j} w_{i j}
$$

Reliable hub network performance measure:

$$
\begin{aligned}
& \left(\sum_{i} \sum_{j} \sum_{k} \sum_{m} w_{i j}\left(1-q_{k}-q_{m k}^{1}+q_{k} q_{m k}^{1}\right) x_{i j k m}\right. \\
& +\sum_{i} \sum_{j} \sum_{k} \sum_{m} \sum_{n} \sum_{l} w_{i j}\left(q_{k} q_{m k}^{2}\left(1-q_{n}\right)\left(1-q_{l n}^{1}\right)\right) x_{i j k m} t_{i j n l} \\
& +\sum_{i} \sum_{j} \sum_{k} \sum_{m \neq k} \sum_{n} w_{i j}\left(q_{k}\left(1-q_{m}\right)\left(1-q_{n m}^{1}\right)\right) x_{i j k m} z_{i n} \\
& \left.+\sum_{i} \sum_{j} \sum_{k} \sum_{m \neq k} \sum_{n} w_{i j}\left(q_{m}\left(1-q_{k}\right)\left(1-q_{n k}^{1}\right)\right) x_{i j k m} z_{j n}\right) / \sum_{i} \sum_{j} w_{i j}
\end{aligned}
$$

According to Equations 46 and 47, we show our test problem performance in Table 3. The Potential Flows column shows the maximum demand on the network that is equal to $\sum_{i} \sum_{j>i} w_{i j}$. As shown in Table 3, our proposed model has a much higher performance than the classical model.

In order to show the efficiency of iterated local search, we compared its performance with CPLEX. We created some examples from the CAB data set. The number of iterations for the search was set at 400 and 1000 for small cases ( 8 and 10 nodes) and larger instances (15 and 25 nodes) respectively. The results for small cases can ensure the validity of the proposed heuristic and shows that the iterated local search outperforms the exact solution approach, considering computational time. In order to get a proper comparison scale, we defined $\Delta$ according to Equation 48.

$\Delta \%=100 \times \frac{C P L E X O F V-I L S O F V}{C P L E X O F V}$ 
Table 3: Optimization results for the selected data set with reliable points

\begin{tabular}{|c|c|c|c|c|c|c|}
\hline & \multicolumn{3}{|c|}{ Test Problem Served demands } & \multicolumn{3}{|c|}{ Potential flows Performance } \\
\hline & & Reliable & Classical & & Reliable & Classical \\
\hline & CAB-10-3-0.8 & 498165.5 & 437312.3 & 499513 & 99.73 & 87.548 \\
\hline & CAB-10-3-0.2 & 498165.5 & 458533.9 & 499513 & 99.73 & 91.796 \\
\hline \multirow[t]{5}{*}{ Without reliable hul } & CAB-10-2-0.8 & 498843.7 & 478395.8 & 499513 & 99.866 & 95.772 \\
\hline & CAB-10-2-0.2 & 499023.5 & 478395.8 & 499513 & 99.902 & 95.772 \\
\hline & CAB-8-3-0.8 & 301806.8 & 274694.7 & 303567 & 99.42 & 90.489 \\
\hline & CAB-8-3-0.2 & 302591 & 261225.7 & 303567 & 99.678 & 86.052 \\
\hline & CAB-8-2-0.8 & 303450.4 & 276896.9 & 303567 & 99.962 & 91.214 \\
\hline \multirow{9}{*}{ With reliable hub } & CAB-8-2-0.2 & 303454.1 & 266739.9 & 303567 & 99.963 & 87.869 \\
\hline & CAB-10-3-0.8 & 499513 & 476510.4 & 499513 & 100 & 95.395 \\
\hline & CAB-10-3-0.2 & 499513 & 473503.9 & 499513 & 100 & 94.793 \\
\hline & CAB-10-2-0.8 & 499513 & 481649 & 499513 & 100 & 96.424 \\
\hline & CAB-10-2-0.2 & 499513 & 481649 & 499513 & 100 & 96.424 \\
\hline & CAB-8-3-0.8 & 303567 & 277283.1 & 303567 & 100 & 91.342 \\
\hline & CAB-8-3-0.2 & 303567 & 277165.3 & 303567 & 100 & 91.303 \\
\hline & CAB-8-2-0.8 & 303567 & 279188.6 & 303567 & 100 & 91.969 \\
\hline & CAB-8-2-0.2 & 303567 & 277415.9 & 303567 & 100 & 91.385 \\
\hline
\end{tabular}

The value of $\Delta$ shows the amount of deviation of iterated local search from the CPLEX solution. Since we restricted the CPLEX run time to one hour, the CPLEX solution may not have been optimum. Therefore, the value of $\Delta$ may be positive or negative where the positive value of $\Delta$ shows that iterated local search finds a better solution than CPLEX. The results are reported in Table 4 and Table 5. 


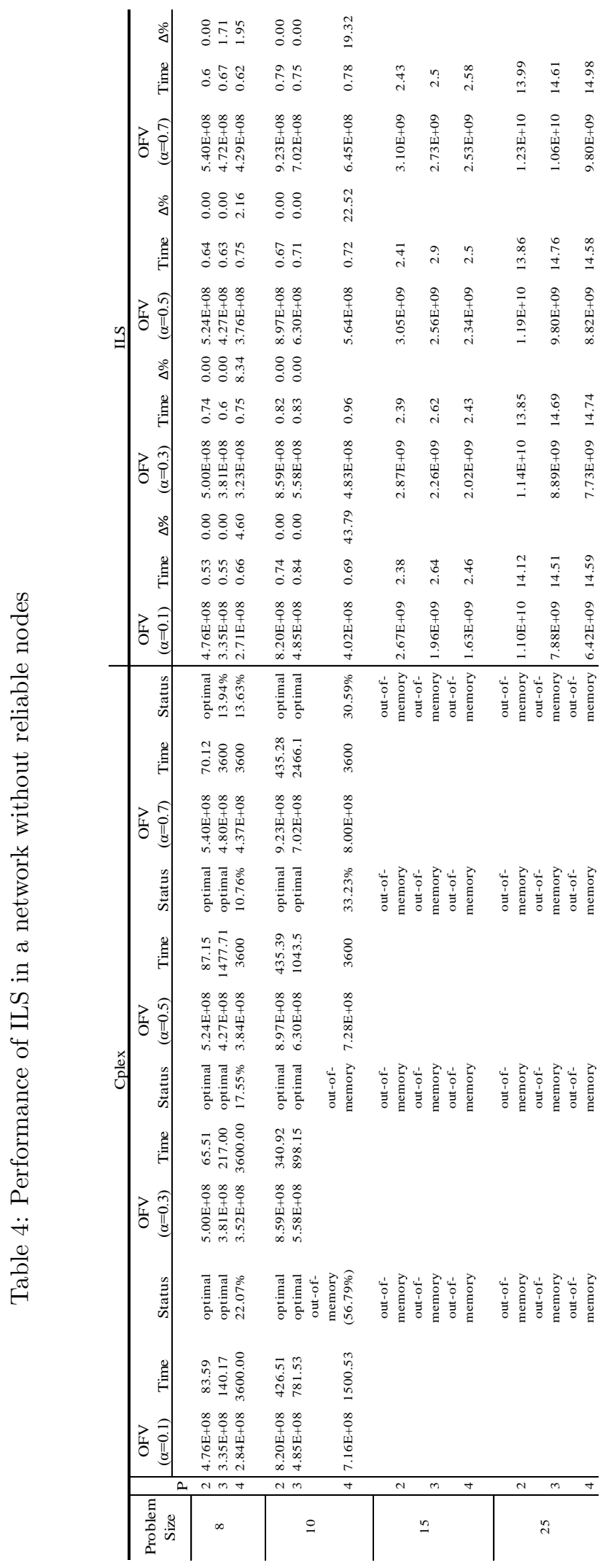




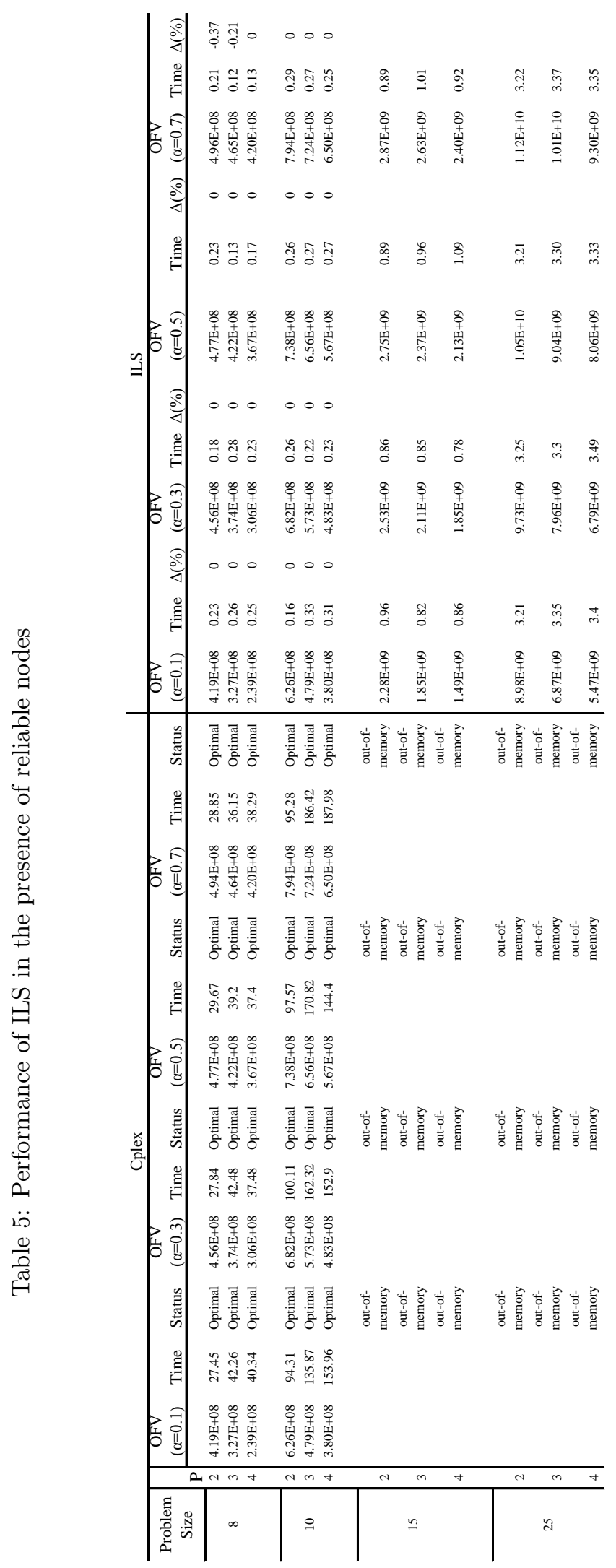


Tables 4 and 5 indicate the solutions obtained from CPLEX, and the best results obtained from ILS in five replications. As reported in Table 4, for some instances (with 8 and 10 nodes), the ILS can reach exact solutions for all small sized instances in a significantly shorter run time comparing with the CPLEX. Moreover, because of the restricted run time of CPLEX, the ILS was able to find a better solution in some of the other instances. For 15 and 25 nodes instances, no feasible solution could be obtained by the CPLEX in the allowed computational time. As it is clear, the ILS could find a near optimal solution in a few seconds. The results show that computational increases by increasing of the problem size. The same results can be considered in the table 5. However, in two small instances (8 nodes), the CPLEX found better solutions (0.37 and $0.21 \%$ better). It is worth mentioning that CPLEX run times are dramatically higher than those of the ILS.

The results shown in Table 4 and Table 5 confirm that applying an iterated local search algorithm reduces the computational time significantly in small cases. In larger instances, this algorithm solved the model in a reasonable time, while CPLEX could not solve the model at all.

\subsection{Algorithm performance on IDA data set}

In this subsection, Iranian Aviation Dataset (IDA) is selected to analyze performance of the proposed algorithm[43]. IDA contains of aviation information of 37 active airports. To adjust this IDA, we considered nodes (3-10-12-14-16-20-22-23-29-32-34) as reliable points and disruption probability of remained nodes are randomly generated from $(0,0.1]$. Since IDA includes more nodes than the $\mathrm{CAB}$, termination criterion is considered as 10000 iterations. The obtained results are reported in Table 6.

Clearly, increasing the number of hubs $(p)$ and decreasing the discount factor reduce the total cost. Moreover, obtained results indicate that by increasing the $p$ computational cost of the algorithm slightly increases (Fig. 7).

\subsection{Model validation analysis}

Consider that all the nodes on the network are reliable. In this case, it is expected that the classical hub network results according to the model proposed in Skorin-Kapov et al.[44] and our proposed model will be the same. We checked that for the case of $C A B-10-3-0.8$ and (9-7-4) were the location of hubs and network cost was 358041878.8 in both models. The result confirms that the proposed model performs as expected. 
Table 6: Computational results for the IDA data set

\begin{tabular}{|c|c|c|c|c|c|}
\hline$p$ & $\alpha$ & Mean OFV $\left(* 10^{8}\right)$ & Best OFV $\left(* 10^{8}\right)$ & Best Location & Average Time $(\mathrm{s})$ \\
\hline 4 & 0.1 & 9.4 & 9.38 & $31-29-23-16$ & 19.39 \\
\hline 4 & 0.4 & 11.4 & 11.31 & $15-23-10-31$ & 19.79 \\
\hline 4 & 0.7 & 13.4 & 12.98 & $23-31-10-34$ & 19.39 \\
\hline 6 & 0.1 & 7.45 & 7.39 & $31-36-16-23-10-29$ & 20.63 \\
\hline 6 & 0.4 & 10.02 & 9.97 & $31-23-34-10-12-14$ & 20.68 \\
\hline 6 & 0.7 & 12.05 & 11.93 & $23-31-34-12-10-20$ & 20.74 \\
\hline 8 & 0.1 & 6.26 & 6.14 & $23-36-31-10-29-30-2-24$ & 22.58 \\
\hline 8 & 0.4 & 9.23 & 9.13 & $23-36-31-10-29-30-2-24$ & 23.59 \\
\hline 8 & 0.7 & 11.8 & 11.73 & $12-30-23-34-10-32-31-20$ & 21.64 \\
\hline
\end{tabular}

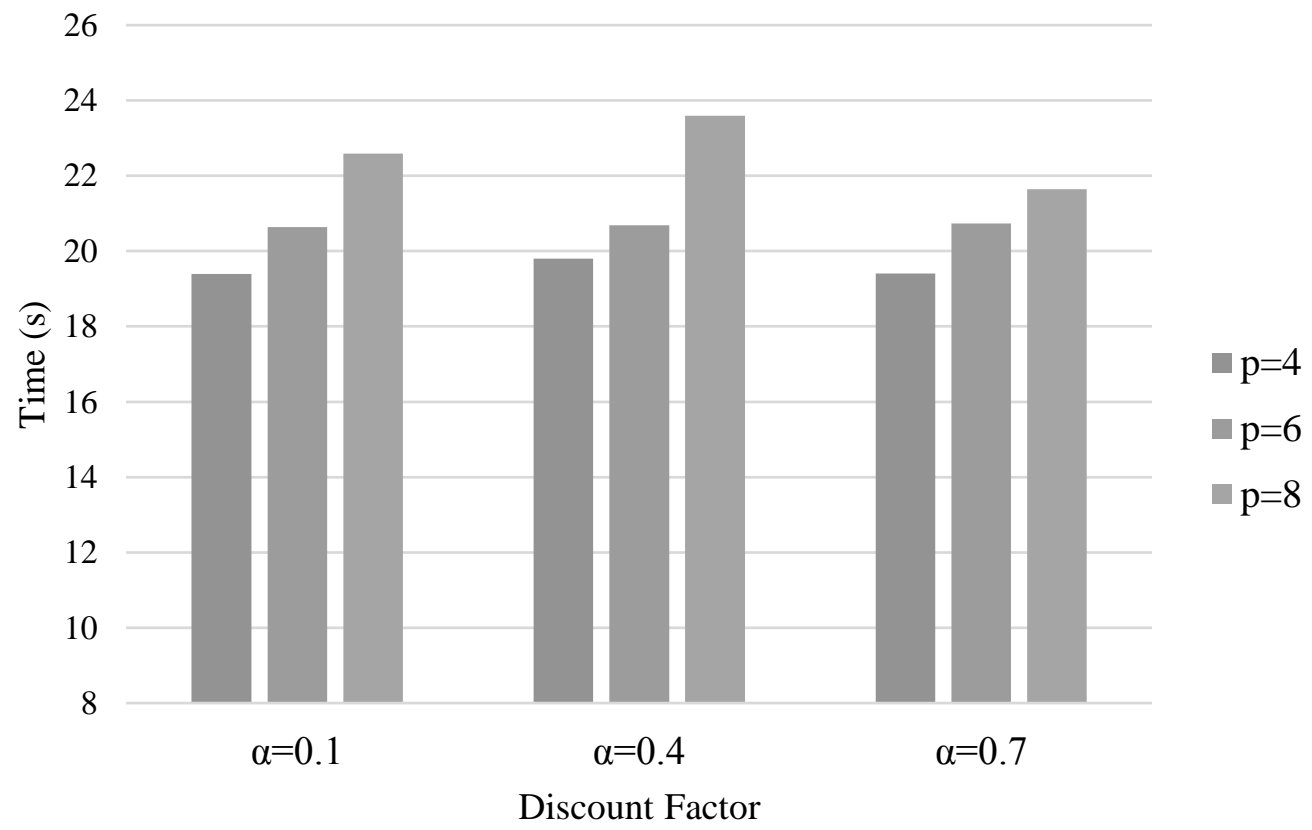

Figure 7: Computational efforts of proposed algorithm for the IDA data set 


\section{Concluding Remarks}

In this paper, hub location problems with potential disruption hubs in network is studied. A new quadratic model is present and the aim of the model is to find the location established hubs and the allocations in normal situation (primal) and also in case of disruption (backup) so that minimize the number unserved flows in case of disruption as a measure of network responsiveness and total network cost based on a lexicographic order. To solve the general bi-objective lexicographic model a two-step optimization approach is needed. However, it is proved that the model can be transform to a classic single objective one by adding a new structure- based constraint. While it may be solved by commercial quadratic integer programming solvers directly, the model is linearized based on three techniques so that it is solved with great efficiency in small-sized problem. The obtained result shows that tight bound linearization technique takes less run time than two other techniques while objective function values of all three techniques are similar. A comparison between classic model and the proposed model in terms of capability in serving flows in normal and disruption situation shows that the proposed model is significantly more efficient that classic one.

Furthermore, the problem is NP-hard and this means that optimal solution of large instances cannot obtained by exact algorithm in a reasonable time. As a result, an efficient heuristic algorithm based on ILS is developed. A comparison of $\Delta$ reveals that ILS outperforms CPLEX in most cases for each test instance. Moreover, the result reveals that CPLEX is highly sensitive to the size of problem its run times are dramatically higher than those of the ILS and it cannot solve the instances with 15 nodes. We check the validity of our proposed model in special cases of the classical hub network, and the results confirm this.

In real world data may not characterize disruption probability sufficiently and an interesting future research direction is to investigate two-stage robust approach can be suggested for future research direction. Moreover, due to

computational complexity of problem, development of new algorithm hybridized with the ILS can be suggested as another future research direction.

[1] R.Z. Farahani, M. Hekmatfar, A.B. Arabani and E. Nikbakhsh. Hub location problems: A review of models, classification, solution techniques, and applications. Comput Ind Eng, vol. 64, no.4, pp. 1096-1109 (2013) 
[2] N. Adler, and N. Hashai. Effect of open skies in the Middle East region. Transp Res Part A, Vol. 39, no. 10, pp. 878-894, 2005.

[3] B. Zhang, Business Insider, 23 Jun 2016. [Online]. Available: http://www.businessinsider.com/winter-storm-jonas-airport-shutdown2016-1.

[4] M. Bashiri, M. Mirzaei and M. Randall, Modeling fuzzy capacitated phub center problem and a genetic algorithm solution, Appl Math Model, vol. 37, no. 5, pp.3513-3525, (2013)

[5] S. A. Alumur, S. Nickel and F. Saldanha-da-Gama, Hub location under uncertainty, Transp. Res. Part B, vol. 46, pp. 529-543, (2012)

[6] I. Contreras, J.-F. Cordeau and G. Laporte, Stochastic uncapacitated hub location, Eur J Oper Res, vol. 212, pp. 518-528, (2011)

[7] T. Ahmadi, H. Karimi, H. Davoudpour and S. Hosseinijou, A robust decision-making approach for p-hub median location problems based on two-stage stochastic programming and mean-variance theory: a real case study, Int J Adv Manuf Technol, vol. 77, pp. 1943-1953, (2015)

[8] S. A. Alumur, B. Y. Kara and O. E. Karasan, The design of single allocation incomplete hub networks, Transp. Res. Part B, vol. 43, pp. 936-951, (2009)

[9] H. Calkk, S. A. Alumur, B. Y. Kara and K. O. E., A tabu-search based heuristic for the hub covering problem over incomplete hub networks, Comput Oper Res, vol. 36, pp. 3088-3096, (2009)

[10] H. Kim and M. E. O'Kelly, Reliable p-Hub Location Problems in Telecommunication Networks, Geogr Anal, vol. 41, pp. 283-306, (2009)

[11] H. Kim, Reliable P-Hub Location Problems and Protection Models for Hub Network Design, (2008)

[12] S. Davari, M. FazelZarandi and I. Turksen, The Fuzzy Reliable Hub Location Problem, in Fuzzy Information Processing Society, Annual Meeting of the North American, (2010)

[13] H. Kim, P-hub protection models for survivable hub network design, J Geogr Syst, vol. 14, no. 4, pp. 437-461, (2012) 
[14] M. P. Scaparra and R. L. Church, A bi-level mixed-integer program for critical infrastructure protection planning, Comput Oper Res, vol. 35, pp. 1905-1923, (2009)

[15] F. Parvaresh, S. Moattar Husseini, S. Hashemi Golpayegany and B. Karimi, Hub network design problem in the presence of disruptions, J Intell Manuf, vol. 25, no. 4, pp. 755-774, (2014)

[16] T. L. Lei, Identifying Critical Facilities in Hub-and-Spoke Networks: A Hub Interdiction Median Problem, Geogr Anal, vol. 45, p. 105-122, (2013)

[17] P. Ramamoorthy, S. Jayaswal, A. Sinha and N. Vidyarthi, Multiple allocation hub interdiction and protection problems: Model formulations and solution approaches, Eur J Oper Res, vol. 270, no.1, pp. 230-245 (2018)

[18] N. Ghaffarinasab and R. Atayi, An implicit enumeration algorithm for the hub interdiction median problem with fortification, Eur J Oper Res, vol. 267, no. 1, pp. 23-39, (2018)

[19] N. Ghaffarinasab and A. Motallebzadeh, Hub interdiction problem variants: Models and metaheuristic solution algorithms, Eur J Oper Res, vol. 267, no. 2, pp. 496-512, (2018)

[20] Y. An, Y. Zhang and B. Zeng, The reliable hub-and-spoke design problem: Models and algorithms, Transp. Res. Part B, vol. 77, pp. 103-122, (2015)

[21] N. Azizi, S. Chauhan, S. Salhi and N. Vidyarthi, The impact of hub failure in hub-and-spoke networks: Mathematical formulations and solution techniques, Comput Oper Res, vol. 65, pp. 174-188, (2016)

[22] M. Mohammadi, R. Tavakkoli-Moghaddam, Siadat, A. and J. Y. Dantan, Design of a reliable logistics network with hub disruptions under uncertainty. Appl Math Model, vol. 40, no. 9, pp. 5621-5642, (2016)

[23] M. Mohammadi, R. Tavakkoli-Moghaddam, A. Siadat and Y. Rahimi, A game-based meta-heuristic for a fuzzy bi-objective reliable hub location problem, Eng Appl Artif Intel, vol. 50, pp. 1-19, (2016)

[24] N. Azizi, Managing facility disruption in hub-and-spoke networks: formulations and efficient solution methods. Ann. Oper. Res., pp.1-27, (2017) 
[25] M. Yahyaei, and M. Bashiri, Scenario-based modeling for multiple allocation hub location problem under disruption risk: multiple cuts Benders decomposition approach, J Ind Eng Int, vol. 13, no. 4, pp. 445-453, (2017)

[26] M. Zhalechian, S. Ali Torabi and M. Mohammadi, Hub-and-spoke network design under operational and disruption risks, Transport Res E Log, vol 109, pp. 20-43, (2018)

[27] B. Rostami, N. Kmmerling, C. Buchheim and U Clausen, Reliable single allocation hub location problem under hub breakdowns, Comput Oper Res, vol. 96, pp.15-29, (2018)

[28] L.V. Snyder, and M.S. Daskin. Models for reliable supply chain network design. In Critical Infrastructure, pp. 257-289. Springer, Berlin, Heidelberg, (2007)

[29] H.R. Lourenco, O.C. Martin and T. Stutzle, Iterated local search: Framework and applications. In Handbook of metaheuristics, pp. 363-397. Springer US., (2010).

[30] M. E. O'Kelly, A quadratic integer program for the location of interacting hub facilities, Eur J Oper Res, vol. 32, pp. 393-404, (1987)

[31] X. He, A. Chen, W. A. Chaovalitwongse and H. X. Liu, An improved linearization technique for a class of quadratic 0-1 programming problems, Optim Lett, vol. 6, pp. 31-41, (2012)

[32] X. He, A. Chen, W. A. Chaovalitwongse and H. Liu, On the Quadratic Programming Approach for Hub Location Problems, in Optimization and logistics challenges in the enterprise, Springer, pp. 211-228, (2009)

[33] B.Y. Kara, Modeling and analysis of issues in hub location problem (Doctoral dissertation, Bilkent University), (1999)

[34] J. Xu, C. Wu, Y. Yin, and W. Lin. An iterated local search for the multi-objective permutation flowshop scheduling problem with sequencedependent setup times. Appl Soft Comput, vol. 52 pp. 39-47, (2017)

[35] P. Vaz Penna, A. Subramanian and L. Ochi, An Iterated Local Search heuristic for the Heterogeneous Fleet Vehicle Routing Problem, J Heuristics, vol. 19, no. 2, pp. 201-232, (2013) 
[36] P. Vansteenwegen and M. Mateo, An iterated local search algorithm for the single-vehicle cyclic inventory, Eur J Oper Res, vol. 237, pp. 802-813, (2014)

[37] T. Song,, S. Liu, X. Tang, X. Peng, and M. Chen. An iterated local search algorithm for the University Course Timetabling Problem. Appl Soft Comput, vol. 68, pp. 597-608, (2018)

[38] A. Subramanian, M. Battarra and C. Potts, An Iterated Local Search heuristic for the single machine total weighted tardiness scheduling problem with sequence-dependent setup times, Int J Prod Res, vol. 52, no. 9, pp. 2729-2742, (2014)

[39] M. Lozano, and C. Garca-Martnez, Hybrid metaheuristics with evolutionary algorithms specializing in intensification and diversification: Overview and progress report, Comput Oper Res, vol. 37, no. 3, pp. 481497, (2010)

[40] J. Brando, Iterated local search algorithm with ejection chains for the open vehicle routing problem with time windows. Comput Ind Eng., vol.120, pp.146-159, (2018)

[41] H. D. Sherali and J. C. Smith, An improved linearization strategy for zero-one quadratic programming problems, Optim Lett, vol. 1, pp. 33-47, (2007)

[42] J. G. Klincewicz, Hub location in backbone/tributary network design: a review, Locat Sci, vol. 6, pp. 307-335, (1998)

[43] H. Karimi and M., Bashiri, Hub covering location problems with different coverage types. Sci. Iran., vol. 18, no.6, pp.1571-1578, (2011)

[44] D. Skorin-Kapov, J. Skorin-Kapov and M. E. O’Kelly, Tight linear programming relaxations of uncapacitated p-hub median problems, Eur J Oper Res, vol. 94, pp. 582-593, (1996) 